The hæmorrhage was so trifling, as merely to require the torsion of one small vessel : the oozing having ceased, six strong hempen sutures were passed through the entire thickness of the denuded surfaces, and tied moderately firm. The first one was applied a few lines in front of the anus, and the last one immediately below the meatus urinarius.

A gum-elastic catheter was introduced into the bladder, and the knees were bound together, after which the patient was placed in bed on her left side. The catheter was allowed to remain for the two first days, but owing to the large size of the urethra part of the urine escaped by the side of the instrument, and coming in contact with the uniting surface produced a good deal of irritation; it was therefore removed, and during the five or six following days it was merely introduced occasionally. The antiphlogistic regimen was strictly adhered to for four or five days, during which cold water was constantly applied to the parts, and the vagina occasionally washed out and cleared of coagulated blood, by means of cold-water injections. An anodyne was given at bedtime, and repeated for three or four nights, to allay irritation and confine the bowels. Two of the sutures were removed on the fourth day, and the other four on the sixth : at the expiration of which, union by the first intention had taken place throughout the whole extent. The bowels were moved on the eighth day, and an occasional dose of aperient medicine was afterwards given.

In the course of three weeks she was allowed to leave her bed, and walk about a little; and on the 30th of September she was able to attend to her usual housework: she can retain her urine for from five to six hours, and can evacuate it voluntarily, and without the slightest inconvenience. The bond of union is very firm, and appears like an elongated perineum, extending from the anus to within a quarter of an inch of the urethra. The finger can be introduced into the vagina with great ease, the parts being very dilatable.

Nov. 20. Although the patient has been exposed to considerable exercise, there is not the least return of the prolapsus: on desiring her to strain violently, a portion of the corrugated anterior paries of the vagina becomes visible, but has not the slightest tendency to protrude externally, although the passage into the vagina is enlarged from the longitudinal contraction of the cicatrix.

In performing this operation, I think it would be better not to attempt the union of the posterior part of the labia, but to leave an opening into the vagina between the bond of union and the frenulum sufficiently large to allow of the discharge of the vaginal mucus and menstrual secretion. By adopting this method the operation would be much facilitated; the most troublesome part of it, and, at the same time, the most painful to the patient, being the dissection required for the removal of the frenulum and the parts in front of the anus. During the treatment, likewise, coagulated blood, and the secretions from the vagina and wounds, could be more easily removed; and in case of considerable inflammation occurring after the operation, cold water could be more readily and continuously applied by means of a syringe. By this modification of the operation, the union of the labia would form a kind of bridge, with two communications into the vagina. The extent of this required, would depend very much upon the case : thus, if there was considerable prolapsus of the bladder and anterior paries of the vagina, it would be necessary to carry the union as far as the meatus urinarius, and the posterior opening might be left large enough to admit the finger. If, on the other hand, the tumour was principally formed by the uterus and posterior wall of the vagina, then it would be advisable to leave the posterior opening just sufficiently large to admit an ordinarysized gum-elastic catheter, and the outer one must be left of considerable extent: care, however, must be taken to make the bond of union of sufficient extent, so as to allow for the subsequent contraction of the cicatrix, otherwise one or other of the openings might become so large, as to allow the inverted mucous membrane gradually to be insinuated through it, and any unusual effort would expose the patient to a return of the con. plaint.

Newcastle-on-Tyne, Nov. 20, 1841.

\section{CASE OF \\ TUBERCULAR DISEASE OF THE KIDNEY.}

By William Henderson, M.D., Edinburgh.

Thomas Hammeton, aged 68, a labourer, has suffered more or less from pain in the lumbar regions, particularly the left, for these last forty years: occasionally during that period, has had nearly total suppression of urine for a day or two, having constant desire, although only able to void a few drops at a time, but always attended with an involuntary dropping, sometimes tinged with blood. During the summer he has complained very much of sickness and pain at stomach, which was often much relieved by vomiting. For two weeks previous to death, he only passed about a tablespoonful of urine per day, which became turbid on the application of heat; sometimes it contained mucus. The catheter was introduced, frequently, during that period, but only brought away a few teaspoonfuls each time. The dropping now had entirely ceased. He suffered very much on the first introduction of the instrument, from strictures of old standing, which he states to have received forty 
years ago by the falling of a chalk cliff at Dover; by which accident he received many other injuries, and dates all his ailments from that period. For the last fourteen days of his life he suffered much from bilions vomiting, and conld retain nothing but a little wine and water, and that only for a short time.

Dissection. - Kidneys much enlarged; right kidney soft, an external ulcer of considerable depth on both sides, the tubuli hard, rounded off, and filling up the crescentic form of its internal border.

Left kidney very soft, of a veal whiteness, all traces of tubuli lost; its whole medullary portion studded with tubercles; its pelvis large, capable of holding a tablespoonful of fluid, and lined throughout with a strong glistening membrane; it was united to the spine and spleen by a scirrhous deposition of a light yellow colour. The liver was considerably enlarged; the spleen was small, shrivelled, and black spotted; the gallbladder was small, and contained a whitish, thick, mucous fluid; the duodenum, at its first turn, was bound firmly down to the right side of the spine by a scirrhous mass which extended downwards into the iliac region; a blank in the left side of this scirrhous wall allowed the bowel to form a sac the size of a large walnut, which held varieties of indigestible matter that he had eaten some weeks previous. The whole duodenum internally was dark-coloured, and contained purulent fluid, formed probably by an ulcer, about the size of a shilling, situated in its mucous coat. The colon was much contracted in calibre, its ascending and descending portions were firmly bound down by old adhesions; a part of its transverse portion of a darkish hue, adhered intimately to the duodenal scirrhous mass : the right half of the pancreas was very soft; the bladder was much contracted, and contained about a tablespoonful of fluid.

Corstorphine, Edinburgh,

Nov. 23, 1841.

\section{POISONING BY "BUTTER OF ANTIMONY."}

\section{To the Editor of The Lancet.}

Sir,-The following case, from the infrequency of similar cases, may, perhaps, be worthy of a place in your columns. Your obedient servant,

John H. Hovghron, M.R.C.S.

Dudley, Nov. 24, 1841.

Nov. 14, half-past ten o'clock, p.m., I was called to see Thomas Ennis, aged ten years, when the following symptoms presented themselves:-Countenance pale ; features collapsed; eyes sunk; pupils dilated and inactive; skin pale and cold; tongue clean ; mouth filled with a thick, tenacious, transparent mucus; nausea; vomiting; pulse about
80, small, but rather firm ; breathing heavy; drowsiness, requiring to be roused before he answers questions, which he then does rationally; he has a severe burning pain in the throat (increased by deglutition), and in a less degree along the course of the osophagus and in the stomach. His mother had bought some " antimony wine" to give him an emetic, apparently without any obvious cause; she poured as much as she thought would be a tablespoonful into a teacup, and gave it to him about two hours before I saw him.

Immediately on drinking it he seemed choked, his features were set, and he was unable to speak for some minutes. He vomited freely, and made frequent ineffectual attempts to do so afterwards; he had then some copious draughts of gruel, which were also returned. He then complained of the pain in his throat, which induced his father to taste the medicine, and finding that it was not "antimony wine" he came for me. I ordered him to drink freely of milk, coffee, white of egg, and gave him a mixture of chalk and water. I applied eight leeches to the epigastrium, and warmth externally ; I also directed his mother not to allow him to sleep long together, for I suspected that he had taken a mixture of some narcotic with a strong acid. I afterwards discovered that it was the "butter of antimony."

15. Countenance flushed; tongue clean; mouth still fills with mucus ; bowels confined; skin hot, rather dry; pulse quick and rather sharp; drowsiness gone off; pain now confined to the throat; no epigastric or abdominal tenderness. He found great relief from the leeches. The fauces are covered with patches of a bright red colour, whilst the intervening parts present a natural appearance. Castor-oil emulsion ; fomentation to the throat; inhalation of the steam of hot water; a simple gargle.

16. Symptoms were all alleviated, but the bowels were still confined. They were then relieved by an enema, after which, by regulating them with castor-oil, he recovered without anything requiring further notice here.

\section{TREATMENT OF UTERINE} H AEMORRHA GE.

Dr. Grattan, of Killeagh, observes," Having directed my attention to the administering of powerful styptics, I commenced by giving tolerably large doses of sulphate of alumina and aromatic sulphuric acid with the most decided benefit: finding that under the use of this medicine the hæmorrhage would become lessened in a few days, I commenced giving a mixture of-

Sulphate of alum, 3iij ;

Sulphate of magnesia, $3 \times$ ij ; Aromatic sulphuric acid, $3 \mathrm{ij}$;

Water, fzxij. M, 